\title{
THROMBOSED DEVELOPMENTAL VENOUS ANOMALY ASSOCIATED WITH CEREBRAL VENOUS INFARCT
}

\author{
Rômulo L. Gama', Mauro Nakayama', Daniel G.F. Távora', \\ Rodrigo C. Bomfim?', Trícia C. Carneiro ${ }^{2}$, Leonardo H.C. Pimentel ${ }^{2}$
}

Developmental venous anomaly (DVA), also known as venous angioma, is becoming the most commonly encountered intracranial vascular malformation in central nervous system (CNS) imaging ${ }^{1,2}$. It is thought that DVA develops during development of the venous system which results in the preservation of primitive embryological medullary veins, draining into a single longer vein ${ }^{3}$. Thrombosis of the collector vein of a DVA is a rare complication leading to cerebral venous infarction (CVI) ${ }^{4}$.

\section{CASE}

A 19 years old woman presented with acute onset of syncope, followed by left side of the body weakness and numbness. These symptoms were followed by consecutive episodes of sei- zures with loss of consciousness. Her admission was done five days after onset of symptoms. She was alert and oriented. Neurological examination demonstrated slight left hemiparesis, hyperactive deep tendon reflexes, impaired superficial sensation and Babinski's sign response on the left side. The Hoffmann's sign was present bilaterally.

MRI revealed a dilated vascular structure at right frontal lobe, filled with hyperintense material on unenhanced T1weighted sequences. It was thought to represent a thrombosed venous collector of a DVA, draining toward the superficial venous system (Fig 1C). Gradient-echo sequences confirmed the endoluminal clot as a hypointense tubular structure due to magnetic susceptibility effect of blood products (Fig 1E). The insular cortex had a mild hyperintensity on unenhanced T1-weight-
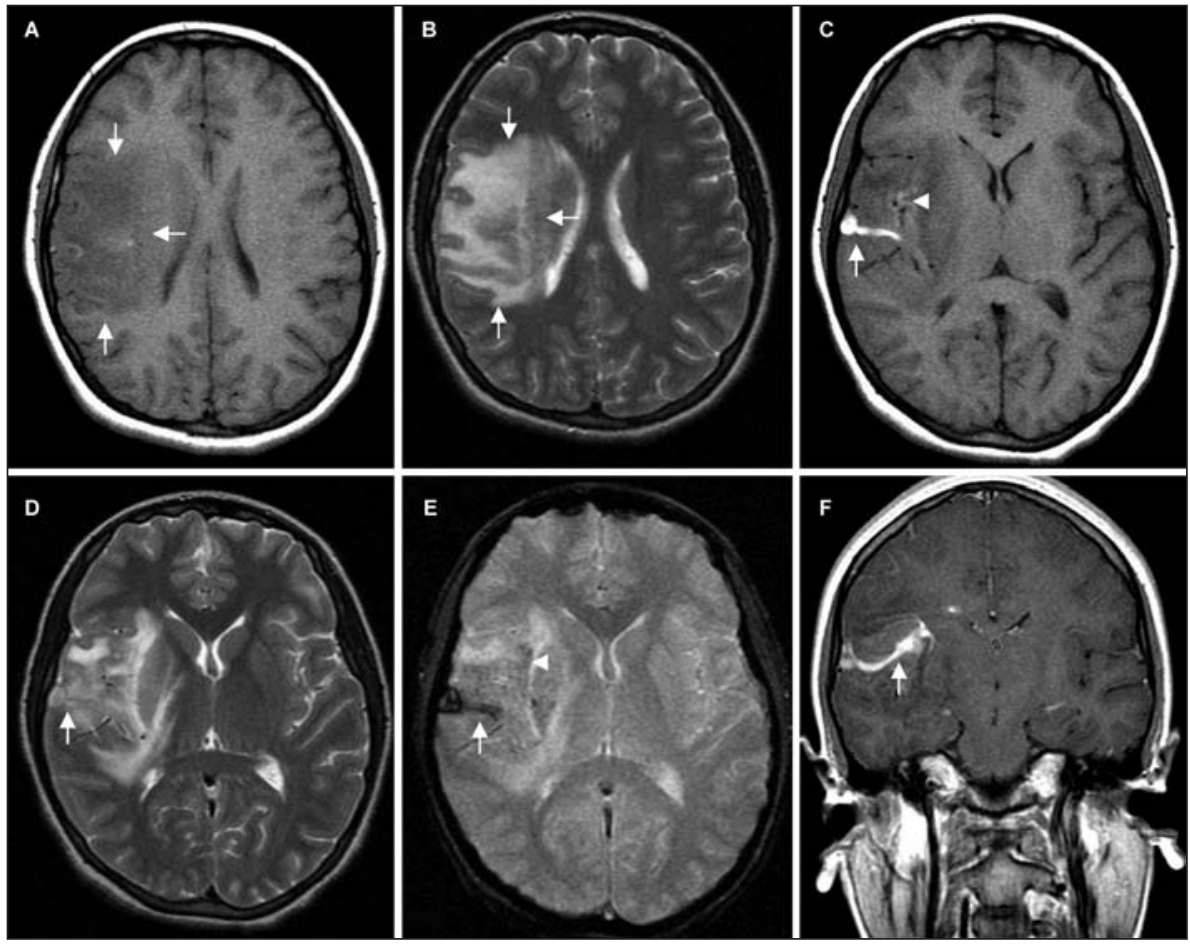

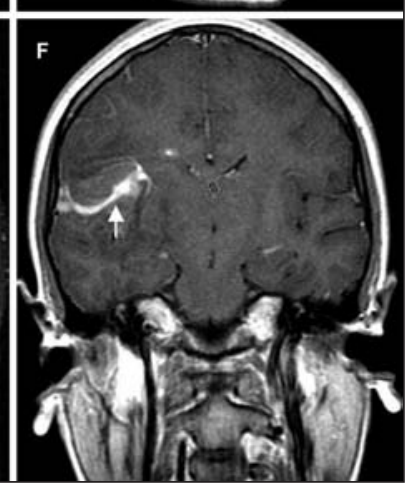

Fig 1. MRI showing thrombosed DVA surrounded by frontoparietal parenchymal edema and tiny intraparenchymal hemorrhage in the right insular cortex. (A and B) T7-weighted and T2-weighted images revealing swollen right frontoparietal area with low signal on $T 7$ (arrows in A) and high signal on T2 (arrows in B). (C) Unenhanced T7weighted image showed hyperintense tubular structure (arrow) and slightly hyperintense area in the insular cortex (arrowhead). (D and E) T2-weighted and Gradient-Echoe T2* images showed high signal (arrow in D) and low signal (arrow in E) in the clot of the thrombosed DVA. (F) Enhanced T7weighted image depicting the typical caput medusae sign of a DVA (arrow).

\section{TROMBOSE DE ANOMALIA VENOSA ASSOCIADA A INFARTO VENOSO CEREBRAL}

Rede Sarah de Hospitais de Reabilitação, Fortaleza CE, Brazil: Departamentos de 'Radiologia e ${ }^{2}$ Lesado Cerebral.

Received 20 February 2008, received in final form 30 May 2008. Accepted 12 June 2008.

Dr. Rômulo L. Gama - Rede Sarah de Hospitais de Reabilitação - Avenida Presidente Juscelino Kubitscheck 4500 - $60867-630$ Fortaleza CE - Brasil. E-mail: romulogama@sarah.br 

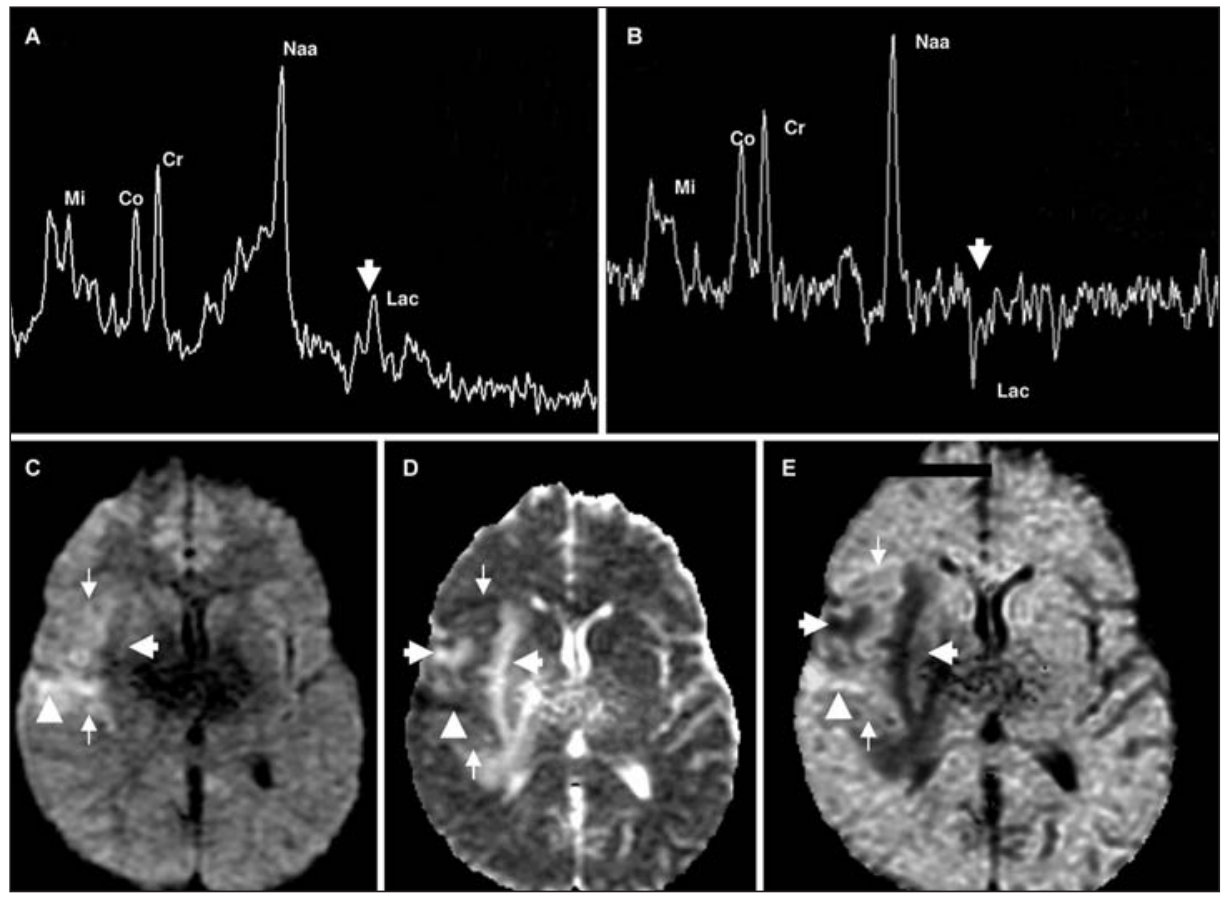

Fig 2. Single voxel ${ }^{7} H-M R S, T E=35 \mathrm{~ms}(A)$ and $T E=144 \mathrm{~ms}(B)$ in the right frontoparietal region showed a preserved NAA peak and a positive doublet peak (A) and inverted doublet peak (B) at 1.31 ppm, indicating lactate peak elevation. DWI (C), ADC map (D) and exponential $A D C$ map (E) at the level of the thrombosed DVA. Endoluminal clot is shown with high signal intensity on DWI and exponential ADC map (arrowhead in C, E) and with low signal intensity on ADC map (arrowhead in D). Cytotoxic edema is depicted as a heterogeneous hyperintense area on DWI and exponential ADC map, with relatively low signal on ADC map (small arrows). Vasogenic edema was predominantly hyperintense on DWI and ADC map and hypointense on exponential ADC map (large arrows).

ed sequences and hypointensity on T2-weighted sequences due to parenchymal hemorrhage in early subacute stage (Fig $1 \mathrm{C}$ and 1D). Adjacent frontoparietal area and basal ganglia showed extensive edema, with hypointense signal on $\mathrm{T1}$ weighted sequences and hyperintense signal on T2-weighted sequences (Fig 1A and 1B). DWI demonstrated high signal in the intravascular clot. Mild hyperintense signal was also detected in the brain parenchyma surrounding the thrombosed collector and in the insular cortex. ADC map in these areas revealed heterogeneously hypointense signal. The remaining edematous areas appeared hyperintense on ADC map, indicating vasogenic edema (Fig 2C and 2D). After administration of intravenous paramagnetic contrast media (Gadolinium), T1- weighted images revealed the typical caput medusae appearance of the DVA (Fig 1F). Single voxel 'H-MRS was performed at the right frontoparietal region. Additional voxel was located at the corresponding area on the left hemisphere for comparison. 'H-MRS spectra were obtained with a short echo time (TE) ( $35 \mathrm{msec}$ ) and an intermediate TE (144 msec). Normal $\mathrm{N}$-acetylaspartate (NAA) peak was found. Spectra also showed a positive doublet peak at $1.31 \mathrm{ppm}$ with short echo time and a corresponding inverted doublet peak at intermediate TE, indicating lactate peak elevation (Fig 2A and 2B).

Anticoagulant therapy with low-molecular-weight heparin was initiated and the clinical scenario improved promptly. Fol- low-up MRI and DWI, obtained 3 months later, showed partial recanalization of the venous collector of the DVA and disappearance of parenchymal edema.

An institutional review board approval was obtained.

\section{DISCUSSION}

Vascular malformations of the CNS are classified by size, location and morphology. The four major pathologic types are capillary telangiectasis, cavernous malformations, DVA and arteriovenous malformations ${ }^{3}$. DVA are the most common vascular anomaly in the CNS, and 50-80\% of DVAs are located in the cerebral hemispheres 5 . DVA have a characteristic appearance on CT and MRI studies. They are rarely detectable without contrast on CT study. After administration of contrast medium the draining veins appear as a linear focuses of enhancement. On brain MRI sequences DVA is depicted as flow void tubular images on both $\mathrm{T} 1$ and $\mathrm{T} 2$ weighted images. After intravenous administration of gadolinium, marked enhancement of the draining and medullary veins occurs, forming the typical caput medusae appearance ${ }^{1-3,5}$.

In our case, MRI revealed abnormal signal intensity involving not only subcortical white matter, but the overly- 
ing cortex adjacent to the DVA. The thrombosed collector vein of the DVA was hyperintense on T1-weighted images and hypointense on gradient-echo $\mathrm{T}^{*}$ weighted images, corresponding to the presence of intravascular $\operatorname{clot}^{6}$.

DWI and ADC map revealed a tiny area of cytotoxic edema at the vicinity of the DVA, surrounded by a larger area of vasogenic edema. In the insular cortex, the presence of hemorrhagic areas might have been responsible for the local decrease in signal intensity in the ADC map, probably as a result of susceptibility effects from blood products. Although venous congestion produced predominantly vasogenic edema, occurrence of cytotoxic edema characterizes the presence of infarcted tissue and underlying CVI. Time interval since the onset of symptoms must be taken into consideration when interpreting $A D C$ map in this case. As a matter of fact, this is an expected phenomenon in the pathophysiology of cerebral venous thrombosis (CVT), caused by congestion of vessels that leads to decreased cerebral blood flow ${ }^{7}$. Thus, decreased $A D C$ in abnormal brain areas associated with CVT may not have the same prognostic value as those associated with pure arterial stroke. Clinical improvement in our patient reinforces the potential for recovery of $\mathrm{CVI}^{8}$.

In our case, a thrombosed DVA was complicated by CVI. 'H-MRS depicted normal NAA peak indicating preserved neuronal viability and it should be considered a good prognostic factor. Another ${ }^{1} \mathrm{H}-\mathrm{MRS}$ finding was elevation of lactate peak, which can indicates cerebral ischemia. However, the detection of lactate does not necessarily denote the presence of infarcted tissue. Increase in lactate peak may represent ischemic tissue at risk of infarction and a potentially reversible condition ${ }^{9}$. Indeed, the patient had a good functional recovery and followup examination showed a total disappearance of the va- sogenic edema and presence of a small remaining area of gliosis.

There is a wide range of etiologies for CVT. Milano et al. described a case associated to prothrombin G20210 mutation $^{10}$. This case report argues against the assumption that DVA is completely benign in nature. MRI not only allows the diagnosis of DVA, but can also rule out associated abnormalities and depict serious complications, such as spontaneous thrombosis of the collector vein with associated hemorrhagic cerebral venous infarction. Currently available physiological MR techniques (DWI and ' $\mathrm{H}-\mathrm{MRS}$ ) can give important prognostic information, especially regarding reversibility of findings and should be included in the imaging work-up of those patients.

\section{REFERENCES}

1. Truwit, C. Venous angioma of the brain: history, significance, and imaging findings. AJR Am J Roentgenol 1992;159(6):1299-1307.

2. Peltier J, Toussaint P, Desenclos C, Gars DL, Deramond H. Cerebral venous angioma of the pons complicated by nonhemorrhagic infarction: case report. J Neurosurg 2004;101:690-693.

3. Töpper R, Jürgens E, Reul J, Armin T. Clinical significance of intracranial developmental venous anomalies. J Neurol Neurosurg Psychiatry 1999;67:234-238.

4. Kim P, Castellani R, Tresser N. Cerebral venous malformation complicated by spontaneous thrombosis. Child's Nerv Syst 1996;12:172-175.

5. Lee C, Pennington A, Kenney CM. MR evalution of developmental venous anomalies: medullary venous anatomy of venous angiomas. AJNR Am J Neuroradiol 1996;17:61-70.

6. Selim M, Fink J, Linfante I, Kumar S, Schlaug G, Caplan LR. Diagnosis of cerebral venous thrombosis with echo-planar $\mathrm{T} 2{ }^{*}$-weighted magnetic resonance imaging. Arch Neurol 2002;59:1021-1026.

7. Favrole P, Guichard JP, Crasssard I, Bousser MG, Chabriat H. Diffusionweighted imaging of intravascular clots in cerebral venous thrombosis. Stroke 2004;35:99-103.

8. Masson C, Godefroy O, Leclerc X, Colombani JM, Leys D. Cerebral venous infarction following thrombosis of the draining vein of a venous angioma (developmental abnormality). Cerebrovasc Dis 2000;10:235-238.

9. Hsu LC, Lirng JF, Fuh JL, Wang SJ, Shyu HY, Liu HC. Proton magnetic resonance spectroscopy in deep cerebral venous thrombosis. Clin Neurol Neurosurg 1998;100:27-30.

10. Milano JB, Arruda WO, Nikosky JG, Meneses MS, Ramina R. Cerebral and systemic venous thrombosis associated to prothrombin G20210 mutation: case report. Arq Neuropsiquiatr 2003;61:1042-1044. 